ESJ Social Sciences

\title{
Análisis bibliométrico sobre energía solar fotovoltaica aplicada en el sector agrícola como alternativa socioambiental
}

\author{
Juan Alexander Armijos Armijos, Ec. Agrop. \\ Eveligh Prado-Carpio, PhD \\ Oliverio Napoleón Vargas Gonzales, Mgs. \\ Javier Yunga- Marquez, Mgs. \\ Universidad Técnica de Machala, Ecuador \\ Abrahan Rodolfo Cervantes-Alava, Mgs. \\ Technical University of Machala, Ecuador
}

\section{Doi:10.19044/esj.2022.v18n1p77}

Submitted: 29 December 2021

Accepted: 26 January 2022

Published: 31 January 2022
Copyright 2022 Author(s)

Under Creative Commons BY-NC-ND

4.0 OPEN ACCESS

Cite As:

Armijos J.A.A., Prado-Carpio E., Vargas Gonzales O.N. \& Cervantes-Alava A.R. (2022). Análisis bibliométrico sobre energía solar fotovoltaica aplicada en el sector agrícola como alternativa socioambiental. European Scientific Journal, ESJ, 18 (1), 77.

https://doi.org/10.19044/esj.2022.v18n1p77

Resumen

La investigación analiza mediante la bibliometría, publicaciones relativas a la energía solar fotovoltaica aplicada en el sector agrícola mundial durante el quinquenio 2017- 2021. La metodología utilizada se ajustó a un proceso de búsqueda y revisión literaria en las principales bases de datos: Redalyc, Science Direct, SciELO y Science.gov; con 30 artículos que tratan sobre el tema, y como resultado se concluye que el mayor número de publicaciones se han realizado en China, con incremento de las publicaciones en el 2021 en Science Direct.

Palabras claves: Análisis bibliométrico, fotovoltaica, sector agrícola, base de datos, socioambiental 


\title{
Bibliometric Analysis of Photovoltaic Solar Energy Applied in the Agricultural Sector as a Socio-Environmental Alternative
}

\author{
Juan Alexander Armijos Armijos, Ec. Agrop. \\ Eveligh Prado-Carpio, PhD \\ Oliverio Napoleón Vargas Gonzales, Mgs. \\ Javier Yunga-Marquez, Mgs. \\ Universidad Técnica de Machala, Ecuador \\ Abrahan Rodolfo Cervantes-Alava, Mgs. \\ Technical University of Machala, Ecuador
}

\begin{abstract}
The research analyzes through bibliometrics, publications related to solar photovoltaic energy applied in the global agricultural sector during the five-year period 2017-2021. The methodology used was adjusted to a process of search and literature review in the main databases: Redalyc, Science Direct, SciELO and Science.gov; with 30 articles dealing with the topic, and as a result it is concluded that the highest number of publications have been made in China, with increased publications in 2021 in Science Direct.
\end{abstract}

Keywords: Bibliometric analysis, photovoltaic, agricultural sector, data base, socio-environmental

\section{Introduction}

El análisis bibliométrico es un procedimiento de cálculo en base a características bibliográficas observadas en la literatura publicada en los círculos científicos y académicos, que ayuda a analizar las características de la actividad científica que incluye la producción y usabilidad de la información. Para el análisis se requiere información bibliográfica completa utilizando una base de datos bibliográfica consistente en un conjunto de registros con información bibliográfica actualizada (Ardanuy, 2012).

Un estudio bibliométrico tiene por objetivo principal evaluar la elaboración de investigaciones científicas para comprender su naturaleza a través de estudios estadísticos cualitativos y/o cuantitativos, con información procesada utilizando modelos matemáticos e instrumentos informáticos para pronosticar o predecir (Rosero \& Vázquez, 2018). Hoy en día el analizar publicaciones crea un eslabón elemental dentro del desarrollo de investigación, que permite apreciar la calidad de conocimiento procesado y el impacto en su entorno (Morocho, Novillo, Cevallos \& Romero, 2019).

La investigación bibliométrica ofrece un amplio abanico de posibilidades para estudiantes y profesionales por su carácter interdisciplinario 
y grado de dificultad, que no excluye el método descriptivo, que, aunque es de menor complejidad no es menos importante (Romaní, Huamaní \& González, 2011).

La energía solar fotovoltaica es una tecnología que genera corriente continua a través de un semiconductor cuando es iluminada por un haz de fotones. Cuando la luz incide en una célula solar, denominado así al elemento fotovoltaico individual, se produce energía eléctrica, que, al momento de apagar la luz, se extingue la electricidad. Las células solares no necesitan cargarse a diferencia de las baterías. Varias células solares han estado en funcionamiento en la tierra o en el espacio durante 30 años (Montes, Fernández, Jiménez, Lecuona, Mellado, Plaza, Ramos \& Sala, 2002).

Los niveles actuales de dependencia de los combustibles fósiles, la necesidad de reducir las emisiones de carbono asociadas con el uso de energía en perspectiva de desarrollar un campo tecnológico nuevo y altamente innovador, hacen que la energía fotovoltaica sea cada vez más atractiva. Estos incentivos han estimulado su crecimiento, haciendo que la industria fotovoltaica esté lista para expandirse. La competencia va en aumento ya que estas nuevas tecnologías están evolucionando permanentemente. Los sistemas solares son un 60\% más baratos hoy que en la década del 90 (Piebalgs \& Potočnik, 2009).

Este tipo de energía puede utilizarse para satisfacer las necesidades energéticas de varias maneras, como el uso de una bomba solar fotovoltaica para regar granjas; un pulverizador y espolvoreador fotovoltaico para protección de plantas; el secador fotovoltaico para trillar materiales y limpiar granos en ausencia de vientos, así como deshidratar las futas y verduras de forma eficaz y eficiente. Todas estas actividades que utilizan energía solar en las explotaciones agrícolas incrementan la producción de cultivos, reducen pérdidas posteriores a la cosecha y mejoran los ingresos (Poonia, Jain, San \& Singh, 2018).

La producción de electricidad por medio de la energía fotovoltaica no genera contaminación, tampoco emite gases efecto invernadero, ni utiliza combustibles fósiles para su funcionamiento (Ishikawa, 2002). Esta energía renovable se considera cada vez más como la opción energética del mañana, porque presenta una solución alternativa y eficaz para minimizar las emisiones de $\mathrm{CO} 2$ y aminorar las compensaciones entre el medio ambiente y el desarrollo energético (Gawande \& Chaudhry, 2019).

La energía solar fotovoltaica ha generado interés social debido al rápido crecimiento de la población y al aumento de la demanda de agua. Se espera que esta alternativa garantice el desempeño económico y ambiental a largo plazo de los sistemas agrícolas, permitiendo cultivos más rentables con una menor degradación de los recursos naturales (Feitosa, Lopes, Andrade, Magalhães \& Freitas, 2021). Así como, de gran utilidad para los lugares de 
difícil acceso a la red eléctrica tradicional, además de los menores costos en comparación al servicio que prestan (Marín, 2019).

Las revistas científicas tienen la función de dar a conocer los avances del conocimiento y ser herramientas de la investigación (Borrego, 2017). Además, sirven como mecanismo de la investigación difundida en los diferentes medios (López-Cózar, Ruiz-Pérez \& Jiménez-Contreras, 2006). Es este entorno, donde los métodos de evaluación bibliométrica poseen particular importancia, ya que cuenta con requisitos básicos como la solidez, autenticidad de la medición, utilización y su reproducibilidad (Gla“nzel \& Moed, 2012).

La presente investigación tiene como objetivo: determinar los principales países con mayor cantidad de investigaciones científicas sobre el tema, el idioma, el año de publicación y la base de datos con mayor número de artículos. El estudio consta de cuatro partes: revisión de literatura, metodología utilizada, presentación de los resultados y conclusiones.

\section{Materiales y métodos}

La metodología utilizada en este estudio, tuvo su sustento en el proceso de revisión de literatura en las bases de datos de Redalyc, Science Direct, SciELO y Science.gov. Las técnicas de observación se realizaron mediante análisis e interpretación, con un enfoque en las variables cuantitativas y cualitativas, con el propósito de determinar el grado de relevancia y originalidad del estudio.

Para este trabajo se utilizó Microsoft Excel 2016, donde los datos recopilados se sometieron al análisis y los resultados representados mediante gráficos estadísticos. El primer grafico indica el país de origen; el segundo, el idioma y año en el que han sido publicadas; y el último, el número de artículos científicos por base de datos.

Es importante señalar que este estudio se centra en la energía solar fotovoltaica aplicada al sector agrícola, y los resultados obtenidos que permiten beneficios en el entorno socioambiental. Para el método de búsqueda de la información se utilizaron las siguientes palabras: "Energía fotovoltaica en la agricultura" o "Photovoltaic energy in agriculture".

\section{Resultados}

Esta sección presenta los resultados del análisis bibliométrico, y responde a las preguntas planteadas en la investigación: ¿qué país alberga la mayor o menor cantidad de publicaciones? ¿en qué idioma y año se han publicado?, y ¿cuál base de datos posee el mayor número de artículos?, todas ellas formuladas en base al objetivo de la investigación. 


\section{Publicación por países de origen}

En el Gráfico 1, se presenta la cantidad de artículos científicos relacionados con el tema de investigación, publicados por el país de origen.

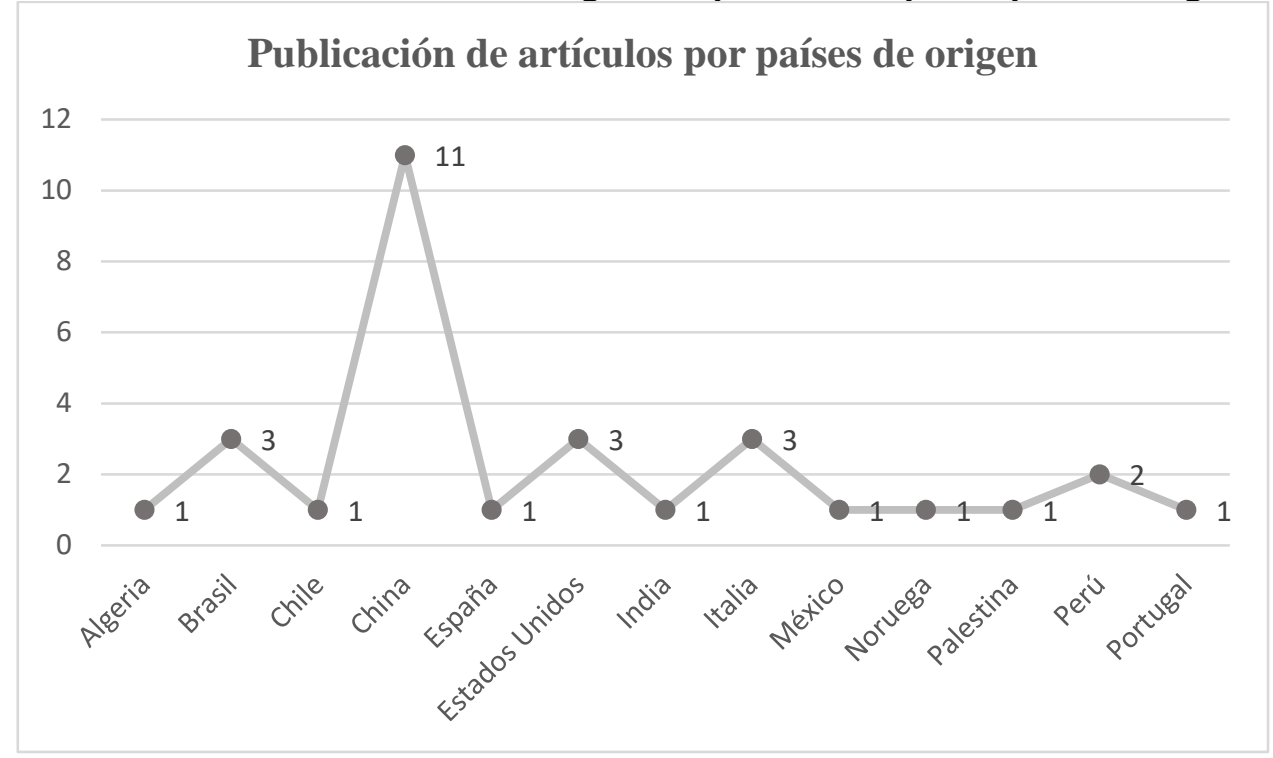

Gráfico 1. Publicación de artículos por países de origen

Fuente: los autores

China es el país con mayor cantidad de publicaciones (11), que representa el 37\% del total de artículos investigados, una diferencia muy notoria contra países como Algeria, Chile, España, India, México, Noruega, Palestina y Portugal, los cuales tuvieron una cantidad menor por año (1), una aportación del $3 \%$.

Es de resaltar que China ha obtenido logros notables en innovación y tecnología en un tiempo récord, lo que ha conmocionado a los gobiernos, empresas y sociedad civil de todo el mundo y muchos análisis se han adelantado a anunciar sobre el surgimiento de un nuevo líder mundial en innovación en las próximas décadas (Estévez, 2019).

Países como Brasil, Estados Unidos e Italia muestran un número bajo de artículos científicos (10\%), pero esto no atribuye a que en otras bases de datos su cantidad sea igual de pequeña, dado que son países con potencial, tanto científico como tecnológico, y pueden tener una gama más amplia de publicaciones.

\section{Publicación de artículos por año}

En el Gráfico 2, se presenta la cantidad de artículos publicados por año, relacionados con el tema de investigación. 


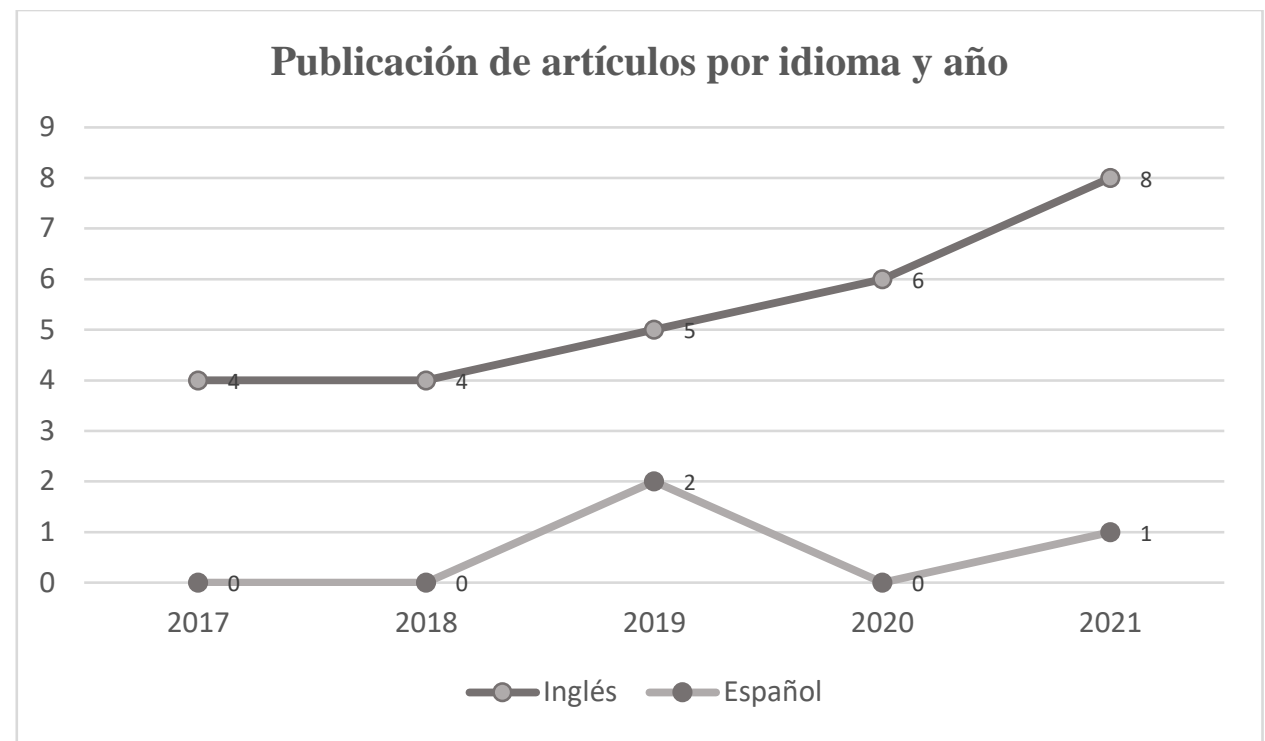

Gráfico 2. Publicación de artículos por idioma y año

Fuente: los autores

Desde el año 2018 ha existido un constante crecimiento de publicaciones en relación a la energía solar fotovoltaica, siendo 2021 el auge para este tema dentro del sector agrícola, donde los países de habla inglesa representan el $90 \%$ del total de las investigaciones.

Este crecimiento no se aprecia en el idioma hispanoamericano, por encontrarse por debajo del nivel de investigación, representando solo el 10\% del total de las investigaciones.

\section{Publicación de artículos por base de datos}

En el Gráfico 3, se presenta la cantidad de artículos científicos disponibles en las bases de datos investigadas. 


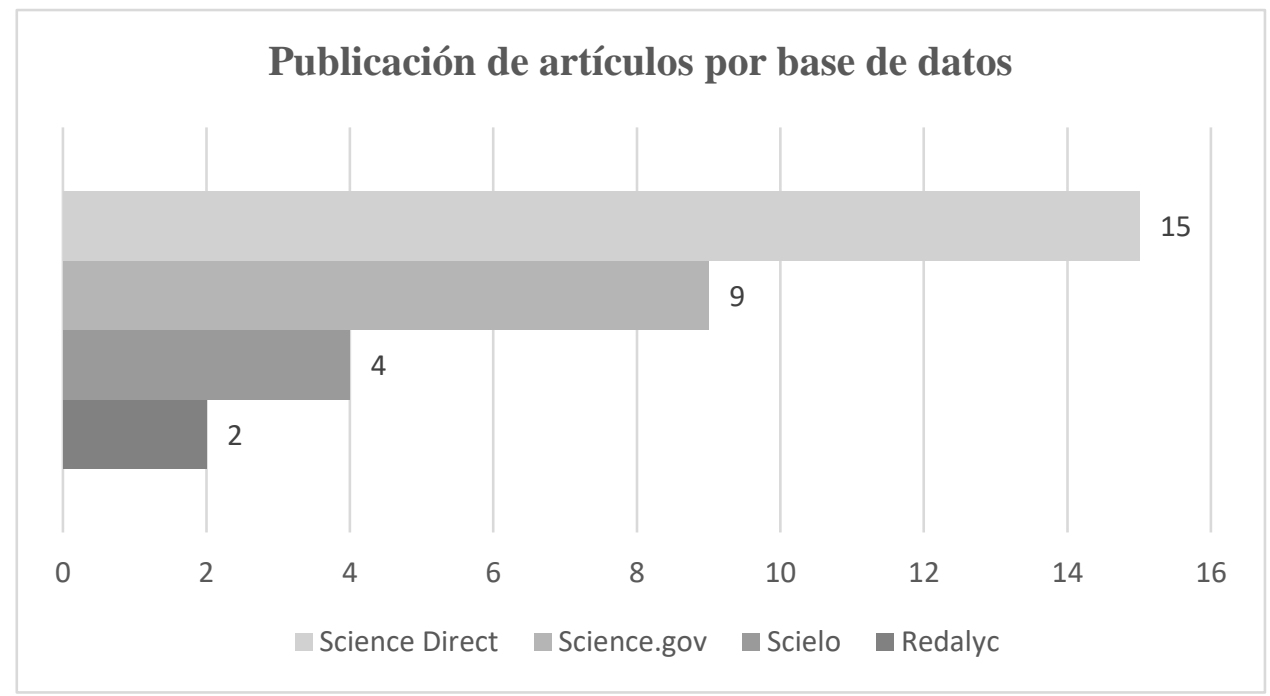

Gráfico 3. Publicación de artículos por base de datos

Fuente: Los autores

Evidentemente, Science Direct, es la base de datos que tiene una mayor aportación en cuanto al tema de la energía solar fotovoltaica (15), representando así el 50\% de todos los artículos analizados.

Así mismo, Science.gov, su aporte es menor (9), que representa el $30 \%$; la diferencia en la cantidad de publicaciones de ambas bases de datos es regular.

Por otro lado, la base de datos Redalyc, cuenta con tan solo (2), constituyendo con el 7\% del total de publicaciones.

Una base de datos consta de varios objetivos, por un lado, apoya la producción de nuevos conocimientos gracias al carácter acumulativo de la ciencia respaldada en trabajos anteriores, y por otro, otorga métricas e informaciones sobre las características de resultados científicos que permiten a los encargados de la ciencia tomar resoluciones informadas (Codina, 2020). La función principal de las bases de datos es asegurar que los investigadores en cada caso tengan la información más relevante para el éxito de su estudio (Codina, 2017).

\section{Conclusiones}

Desde el año 2018 ha existido un constante crecimiento de la investigación fotovoltaica en la agricultura, demostrando así, la relevancia de las energías renovables en este sector y más en la actualidad donde son una alternativa frente a las energías convencionales.

Se observa que la mayor cantidad de aportes científicos se realiza en el idioma inglés (90\%), además de que China es el país que lleva el primer 
puesto, esto debido a que destina mayores recursos en investigación y desarrollo.

Se identificó que la base de datos Science Direct, abarca numerosa información sobre este tipo de energía renovable (50\%), esto debido a que es la base de datos más importante actualmente, y que a diferencia de otras plataformas no requiere suscripción previa.

Las diferentes bases de datos por medio de sus editoriales, permiten que la comunidad académica y científica se beneficie de las múltiples investigaciones y descubrimientos llevados a cabo, permitiendo así el desarrollo de la sociedad en el ámbito científico.

Es muy importante que las energías fotovoltaicas sean consideradas de alta prioridad en los países dedicados al sector agropecuario, pues, al modernizarse y utilizarla, incentivarían la competitividad y sostenibilidad tanto ambiental, económica y social.

Lo que se investiga y no está escrito, o está escrito y no se publica, equivale a no investigación; en este caso, la coherencia se pierde con el tiempo y también la cantidad de información de investigadores y equipos científicos. Por lo tanto, los investigadores están obligados a publicar los resultados de sus investigaciones y a dar gracias por el apoyo financiero de agencias gubernamentales o universidades (Palomares, 2019).

\section{Agradecimientos}

Agradezco al Dr. José Roberto Paladines, investigador jubilado de la "Universidad Técnica de Machala" y Dra. María de Lourdes Olivo por sus orientaciones en este trabajo.

\section{References:}

1. Ardanuy, J. (2012). Breve introducción a la bibliometría. Barcelona: Departament de Biblioteconomia i Documentació.

2. Borrego, Á. (2017). La revista científica: un breve recorrido histórico. Revistas científicas: situación actual y retos de futuro, 19-34.

3. Codina, L. (2017). Investigación con bases de datos. Estructura y Funciones de las Bases de Datos Académicas Análisis de Componentes y Estudio de Caso. Barcelona: Departamento de Comunicación. Grupo DigiDoc.

4. Codina, L. (2020). Estructura y funciones de las bases de datos académicas . Recuperado el 05 de noviembre de 2020, de https://www.lluiscodina.com/bases-de-datos-academicas-registros/

5. Estévez, A. B. (2019). China como líder innovador, entre el éxito y las dudas. Real Instituto Elcano.

6. Feitosa, E. d., Lopes, F. B., Andrade, E. M., Magalhães, A. C., \& Freitas, C. A. (2021). Environmental impact of different agricultural 
production systems. Ciência Agronômica, 52. doi:DOI: 10.5935/18066690.20210003

7. Gawande, A., \& Chaudhry, P. (2019). Environmental and social impacts of solar energy: a view point with reference to India. Jharkhand Journal of Development and Management Studies, 17(2), 8115-8125.

8. Gla"nzel, W., \& Moed, H. F. (2012). Opinion paper: thoughts and facts on bibliometric. Scientometrics. doi:DOI 10.1007/s11192-012-0898-z

9. Ishikawa, T. (2002). Grid-connected photovoltaic power systems: survey of inverter and related protection equipments. Japan.

10. López-Cózar, E. D., Ruiz-Pérez, R., \& Jiménez-Contreras, E. (2006). La Edición de Revistas Científicas Directrices, Criterios y Modelos de Evaluación. Granada: EC3: Evaluación de la Ciencia y de la Comunicación Científica.

11. Marín, L. A. (2019). Uso de la energía solar en la agricultura. Porvenir S.A.

12. Montes, J. I., Durán, A. F., Suárez, C. J., Ribot, A. L., García, F. M., Fernández, J. F., . . Pano, G. S. (2002). Energía Solar Fotovoltaica. En G. NAP, Energía Solar Fotovoltaica. Madrid.

13. Morocho, J. I., Novillo, J. J., Cevallos, H. V., \& Romero, H. C. (abril de 2019). Análisis bibliometrico sobre optimización multiobjetivo con progrmación de metas aplicadas en el sector agropecuario. Observatorio de la Economía Latinoamericana. Obtenido de https://www.eumed.net/rev/oel/2019/04/programacion-metasagropecuario.html

14. Palomares, S. G. (2019). Importancia de publicar artículos científicos. MasScience, 19, 1-4.

15. Piebalgs, A., \& Potočnik, J. (2009). Photovoltaic Solar Energy Development and curret researh. Belgium: European Communities.

16. Poonia, S., Jain, D., Santra, P., \& Singh, A. (September de 2018). Use of solar energy in agricultural production and processing. Indian Farming , 68(09), 104-109.

17. Romaní, F., Huamaní, C., \& González, G. (2011). Estudios bibliométricos como linea de investigación en las ciencias biomédicas: una aproximación para el pregrado (Vol. (14)1). Lima.

18. Rosero, J. M., \& Vázquez, N. C. (2018). Análisis bibliométrico sobre la producción de artículos científicos relacionados con rutas turísticas en (Redalyc) en el periodo 2005 - 2017). Sathiti: sembrador, 13(1), 8192. doi:https://doi.org/10.32645/13906925.503 